\title{
Analytical modeling of oxide-based bipolar resistive memories and complementary resistive switches
}

\author{
Stefano Ambrogio, Student Member, IEEE, Simone Balatti, Student Member, IEEE, David C. Gilmer, \\ Daniele Ielmini, Senior Member, IEEE
}

\begin{abstract}
To allow for novel memory and computing schemes based on the resistive switching memory (RRAM), physicallybased compact models are needed. This work presents a new analytical model for $\mathrm{HfO}_{2}$-based RRAM, relying on a simplified description of the conductive filament (CF) in terms of its diameter and gap length. The set and reset operations are described by $C F$ growth and gap opening, respectively, activated by the local field and temperature. The analytical model is then used to describe the switching dynamics in the complementary resistive switch (CRS), consisting of an antiserial connection of two resistive devices. The impact of the gap resistivity on the CRS characteristics is discussed, highlighting the trade-off between off-state leakage and set/reset window.
\end{abstract}

Keywords: resistive switching, ion migration, crossbar array, metal insulator transition, resistive-switching random access memory, complementary resistive switching (CRS), (RRAM).

\section{INTRODUCTION}

Recently, resistive switching memory (RRAM) has emerged as a promising alternative to Flash memories for high density data storage [1]-[3]. To understand the scaling limits of this technology, physical models have been developed to describe the set/reset processes and the electrical transport phenomena in the conductive filament (CF) [4]-[10]. On the other hand, the simulation of RRAM circuits such as memory arrays and neuromorphic systems requires analytical models for implementation in Spice or Verilog-A [4], [11]-[13]. This work presents an analytical model for set/reset transitions in oxidebased bipolar RRAM devices. The model is an extension of a previous analytical model [11], which was improved to take into account the different dynamics of the set and reset transition that were recently pointed out by experiments [14] and numerical simulations on $\mathrm{HfO}_{x}$ RRAM [10]. The model is applied to account for the switching characteristics of the complementary resistive switch (CRS), consisting of two antiserially connected RRAM devices. After explaining the CRS characteristics based on the sequential switching of the two individual RRAMs, we discuss CRS optimization by reduction of the off-state leakage and its impact on the read window.

S. Ambrogio, S. Balatti and D. Ielmini are with the Dipartimento di Elettronica, Informazione e Bioingegneria and Italian Universities Nanoelectronics Team (IU.NET), Politecnico di Milano, piazza L. da Vinci 32, 20133 Milano, Italy (e-mail daniele.ielmini@polimi.it). D. C. Gilmer is with the Front-End Process and Emerging Technologies, SEMATECH, Austin, TX 78741 USA (e-mail: david.gilmer@sematech.org). This work was supported in part by Intel under Project 55887 and in part by the Fondazione Cariplo under Grant 2010-0500.
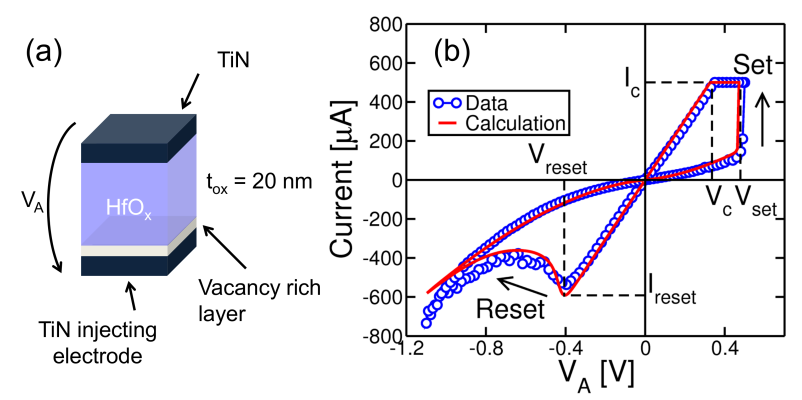

Fig. 1. Schematic representation (a) and measured and calculated $I-V$ curves (b) for a RRAM device showing positive abrupt set and negative analog reset transitions. The current was limited to compliance $I_{C}=500 \mu \mathrm{A}$ during set transition.

\section{EXPERIMENTAL CHARACTERISTICS}

Fig. 1a shows the RRAM structure considered in this work, consisting of a 20-nm thick $\mathrm{HfO}_{2}$ switching layer with TiN bottom and top electrode. The oxide layer had a non-uniform composition profile, with a lower concentration of oxygen close to the bottom electrode, thus causing the presence of a high local concentration of oxygen vacancies, serving as a reservoir for defect injection during filament forming and set. For this reason, the bottom electrode will be referred to as the injecting electrode in the following. The nonuniform distribution was achieved by an additive approach, where the initial oxygen-deficient layer was oxidized on the top-electrode side to increase the oxygen content [15]. The asymmetric structure of the RRAM dictates the polarity for set and reset operations, where set transition takes place under positive voltage $V_{A}$ applied to the injecting electrode, while reset transition is achieved under negative $V_{A}$. Fig. 1b shows the measured $I-V$ curve for a RRAM device, where the current entering the injecting electrode is plotted as a function of $V_{A}$. The device was preliminarily formed at $V_{A}=1 \mathrm{~V}$. The $I-V$ curve displays abrupt set transition for $V_{A}>0$ and gradual reset transition for $V_{A}<0$. During set transition, the current is limited by a compliance current $I_{C}=500 \mu \mathrm{A}$ to limit the size of the $\mathrm{CF}$. The voltage across the $\mathrm{CF}$ reaches a characteristic value $V_{C} \approx 0.4 \mathrm{~V}$ after set transition, as a result of the voltage driven kinetics of ion migration [14]. The reset voltage $V_{\text {reset }}$ in correspondence of the onset of the reset transition is approximately equal to $V_{C}$ since set and reset processes are both dictated by the same ion migration kinetics. As a result, the reset current $I_{\text {reset }}$ is almost equal to $I_{C}$, in agreement with previous data [14] and modeling results [10], [11]. 


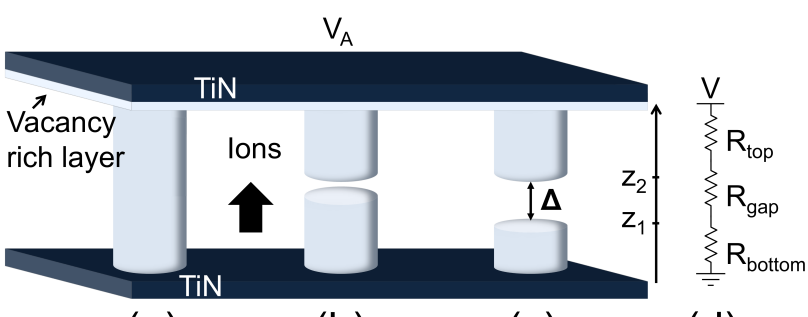

(a)

(b)

(c)

(d)

Fig. 2. Schematic illustration of the reset model. Reset transition is due to the opening of a gap $\Delta$ from the centre of the $\mathrm{CF}$, as evidenced by the steps (a) for a complete CF, (b) and (c) for increasing gap lengths. The circuit topology used in the model is shown in (d).

TABLE I

MODEL PARAMETERS

$\begin{array}{lcr}\text { Activation energy }(\mathrm{F}=0) & \mathrm{E}_{A} & 1.2 \mathrm{eV} \\ \text { Oxide resistivity }(\mathrm{F}=0) & \rho_{o x} & 8.5 \mathrm{~m} \Omega \mathrm{cm} \\ \text { CF resistivity } & \rho_{m} & 270 \mu \Omega \mathrm{cm} \\ \text { Pre-exponential factor } & \mathrm{A} & 300 \mathrm{~ms}^{-1} \\ \text { Non-linearity coefficient } & \gamma & 55 \mathrm{nmV}^{-1} \\ \text { CF thermal conductivity } & \mathrm{k}_{t h, m} & 23 \mathrm{Wm}^{-1} \mathrm{~K}^{-1} \\ \text { Oxide thermal conductivity } & \mathrm{k}_{o x} & 0.68 \mathrm{Wm}^{-1} \mathrm{~K}^{-1} \\ \text { Characteristic length } & \Delta_{\text {eff }} & 10.5 \mathrm{~nm}\end{array}$

\section{THE ANALYTICAL MODEL}

Set and reset processes are interpreted in terms of ion migration, inducing the formation of a gap during reset, responsible for the resistance increase, and a filamentary growth during set, responsible for resistance decrease. The interpretation of set and reset processes originates from the analysis of the experimental data of $V_{\text {reset }}$, showing that different resistance values in set states correspond to different cross-section areas in a continuous $\mathrm{CF}$, while different resistance values in reset states correspond to different gap lengths in an interrupted $\mathrm{CF}$ [14]. These results were corroborated by simulation results of a numerical model based on the migration of ionized defects accelerated by the local temperature and electric field [10]. The same concepts were adopted in a similar analytical model in the literature [4].

\section{A. Reset model and simulations}

Fig. 2 schematically shows the reset process, where the migration of positively ionized defects (oxygen vacancies and/or metallic $\mathrm{Hf}$ or $\mathrm{Ti}$ from the vacancy-rich layer) driven by the electric field and activated by temperature leads to the formation and growth of a depleted gap along the CF. The injecting electrode is shown at the top side in agreement with Fig. 1a. The initial condition is the full set state, where the continuous $\mathrm{CF}$, consisting of segregated metallic Hf in the model, has an idealized cylindrical shape. The negative $V_{A}$ induces ion migration toward the injecting electrode, causing depletion of the CF. The temperature profile along the CF can be obtained analytically solving the 1D steady-state Fourier equation:

$$
k_{t h} \frac{d^{2} T}{d z^{2}}+J^{2} \rho=0
$$

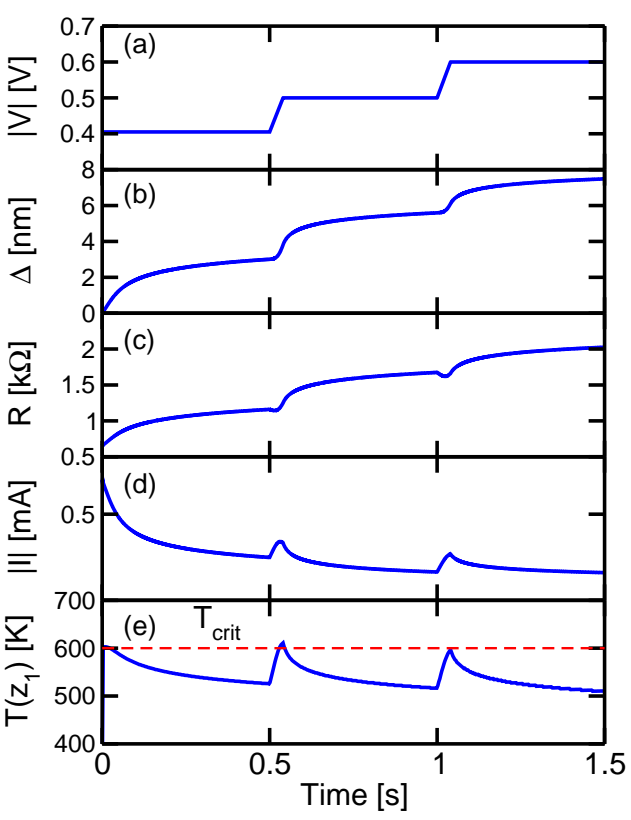

Fig. 3. Calculated voltage $V$, (a), gap length $\Delta$, (b), resistance $R$, (c), current $I,(\mathrm{~d})$, and temperature at the $T\left(z_{1}\right)$ edge, (e), as a function of time. Application of a voltage causes an increase of the gap $\Delta$, leading to a $\mathrm{CF}$ cooling.

where $\mathrm{z}$ is the space coordinate along the $\mathrm{CF}(\mathrm{z}=0$ at the injecting electrode and $t_{o x}=20 \mathrm{~nm}$ is the oxide thickness and the total CF length), $k_{t h}$ is the thermal conductivity, $\rho$ is the resistivity and $J$ is the current density. For the full set state, Eq. (1) was solved using the parameters of metallic Hf, namely $k_{t h}=k_{t h, m}=23 \mathrm{Wm}^{-1} \mathrm{~K}^{-1}$, as for bulk Hafnium, and $\rho=\rho_{m}=270 \mu \Omega \mathrm{cm}$, which is higher than the bulk Hf value to take into account enhanced scattering in the nanoscaled $\mathrm{CF}$ [11], [16]. Parameters $\rho_{m}$ and $k_{t h, m}$ are shown in Tab. I with other modeling parameters. Eq. (1) is solved with boundary conditions $\mathrm{T}(0)=\mathrm{T}\left(t_{o x}\right)=\mathrm{T}_{0}=300 \mathrm{~K}$, where the top and bottom contacts are considered as ideal heat sinks. For the full set state, the solution gives a parabolic profile $\mathrm{T}(\mathrm{x})$ with the maximum temperature in the middle of the $\mathrm{CF}$ at $\mathrm{z}=t_{o x} / 2$ :

$$
T_{\max }=T_{0}+\frac{J^{2} \rho t_{o x}^{2}}{8 k_{t h}}
$$

As $T_{\max }$ in Eq. (2) reaches the critical temperature for ion migration in the timescale of the experiment, a depleted gap starts to form in correspondence of $t_{o x} / 2$, as shown in Fig. $2 \mathrm{~b}$. The resistance correspondingly increases, thus marking the onset of the reset transition at $V_{\text {reset }}$. Reset leads to the rupture of the CF in two filament stubs separated by a gap of length $\Delta$. Further increase of the voltage results in an increase of $\Delta$ by migration of ionized defects from one filament stub to the other toward the injecting electrode. Ion migration thus increases the defect density at the injecting electrode side, while the other filament stub is consumed, in agreement with numerical simulation results [10]. The increase of $\Delta$ is modeled by the Arrhenius law:

$$
\frac{d \Delta}{d t}=A e^{-\frac{E_{A}}{k T\left(z_{1}\right)}},
$$




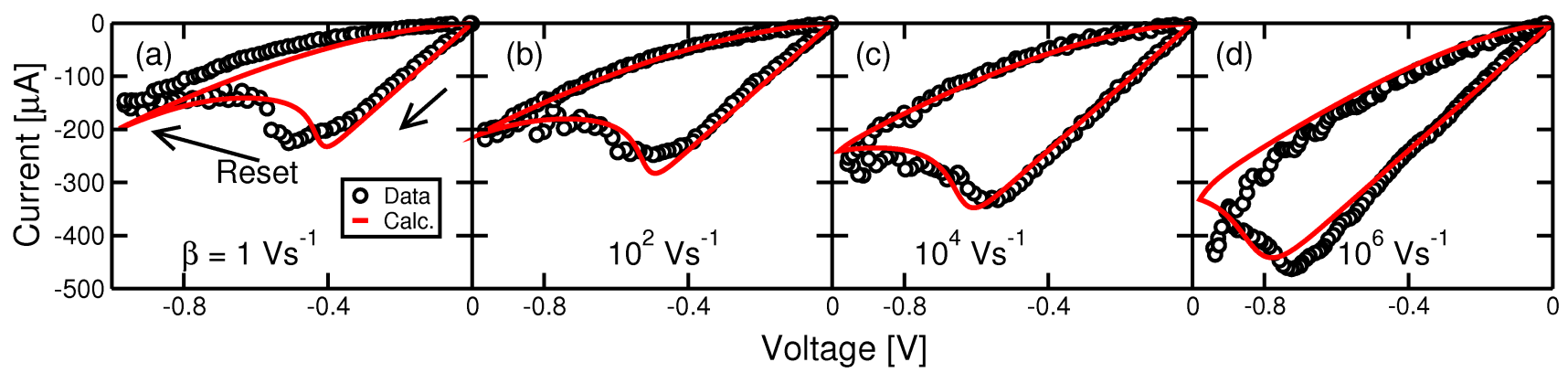

Fig. 4. Measured and calculated $I-V$ curves in the reset transition. Current compliance $I_{C}$ in the previous set process was always fixed at $200 \mu \mathrm{A}$. Results are shown for different sweep rates, namely $\beta=1$ (a), $10^{2}$ (b), $10^{4}$ (c) and $10^{6} \mathrm{Vs}^{-1}$ (d).

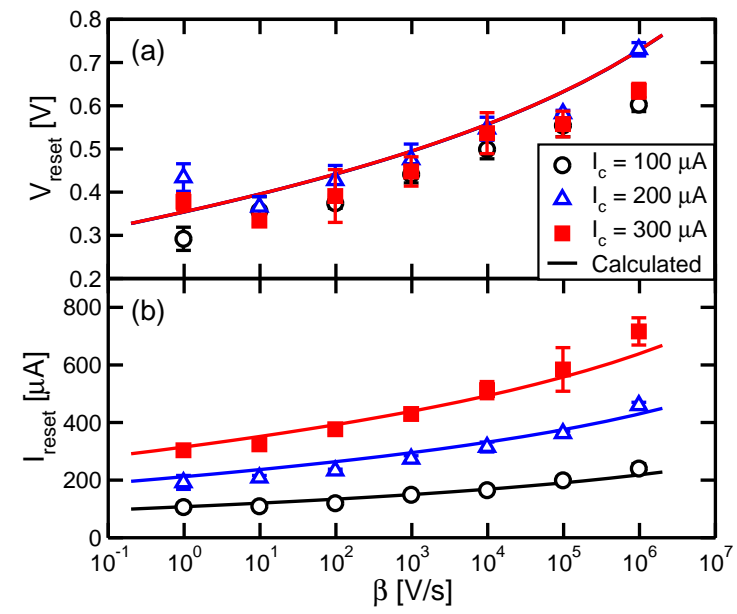

Fig. 5. Measured and calculated $V_{\text {reset }}$ (a), and $I_{\text {reset }}$ (b) as a function of the sweep rate $\beta\left(\mathrm{Vs}^{-1}\right)$. Results are shown for $I_{C}=100,200$ and $300 \mu \mathrm{A}$.

where $\mathrm{A}=300 \mathrm{~ms}^{-1}$ is a pre-exponential factor, $k$ is the Boltzmann constant, $T\left(z_{1}\right)$ is the temperature in correspondence of the ion-injecting stub edge (see Fig. 2c) and $E_{A}$ is the energy barrier for ion migration. The latter is lowered due to Poole-Frenkel effect by the electric field in the gap, as expressed by:

$$
E_{A}=E_{A 0}-\alpha q V_{g a p},
$$

where $\alpha=0.05$ is the barrier lowering factor, $E_{A 0}=1.2 \mathrm{eV}$ is the energy barrier at zero field, $q$ is the unit charge and $V_{g a p}$ is the voltage drop across the gap. The temperature profile in the fragmented $\mathrm{CF}$ is calculated using Fourier equations as in Eq. (1), but solved separately in the three domains (top stub, gap, bottom stub) with appropriate boundary conditions and suitable values for parameters $k_{t h}$ and $\rho$. The thermal conductivity and resistivity in the metallic stubs were assumed equal to $k_{t h, m}$ and $\rho_{m}$, respectively, while the $\rho$ in the gap is given by:

$$
\rho_{g a p}=\frac{\rho_{o x}}{1+\gamma F}
$$

Where $\gamma$ is a constant, $F$ the local electric field and $\rho_{o x}$ is the zero-field gap resistivity. Eq. (5) is a first-order expansion

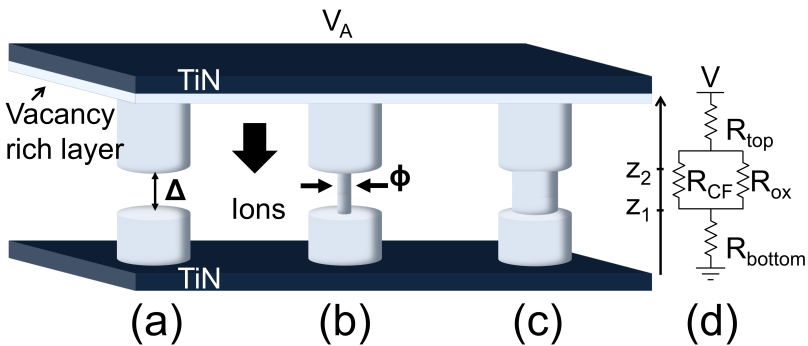

Fig. 6. Schematic illustration of the set model. Set transition is due to the nucleation and growth of a sub-CF $\phi$ in the oxide layer, as evidenced by the steps (a) for a full reset state, (b) and (c) for increasing sub-CF $\phi$. The circuit topology used in the model is shown in $(d)$.

of the Poole conduction law $\rho \propto \exp (-\mathrm{F} \Delta \mathrm{z} /(2 \mathrm{kT}))$ [17], where $\Delta \mathrm{z}$ is the average distance between electron traps. For simplicity, the parameter $\gamma=\mathrm{z} /(2 \mathrm{kT})$ in Eq. (5) was assumed to be a constant, namely $\gamma=55 \mathrm{~nm} / \mathrm{V}$. Eq. (5) accounts for the nonlinear conduction characteristics in the reset state. The thermal conductivity $k_{\text {gap }}$ in the gap was changed continuously with the gap length, from the metallic value $k_{t h, m}$ for $\Delta=0$ to the bulk- $\mathrm{HfO}_{2}$ value $k_{o x}=0.68 \mathrm{Wm}^{-1} \mathrm{~K}^{-1}$ [18] for a characteristic $\Delta_{\text {eff }}=50 \%$ of the thickness. The $\Delta$-dependent $k_{t h}$ reflects the change of defect concentration in the gap for variable length, where an extremely short gap displays a relatively large average concentration of defects, whereas a long gap can be considered to be almost fully depleted [10]. In the model, the variable $k_{t h}$ was achieved with a power law given by:

$$
k_{g a p}(\Delta)=k_{t h, m}+1-\left(1+k_{t h, m}-k_{o x}\right)^{\frac{\Delta}{\Delta_{e f f}}} .
$$

Fig. 1b shows the calculated $I-V$ curves obtained by the model with the parameters in Tab. I, where the reset transition clearly displays a gradual increase of resistance. The model describes the top, bottom stubs and gap as series resistances as shown in Fig. 2d. This gradual increase of resistance can be understood by a negative feedback effect, where ion migration leads to an increase of $\Delta$, which in turn reduces the temperature $T\left(z_{1}\right)$ at the origin of ion migration. As a result, the depletion process is soon interrupted, and a higher voltage is needed to further induce an increase of $\Delta$. The thermal negative feedback is explained by calculations in 


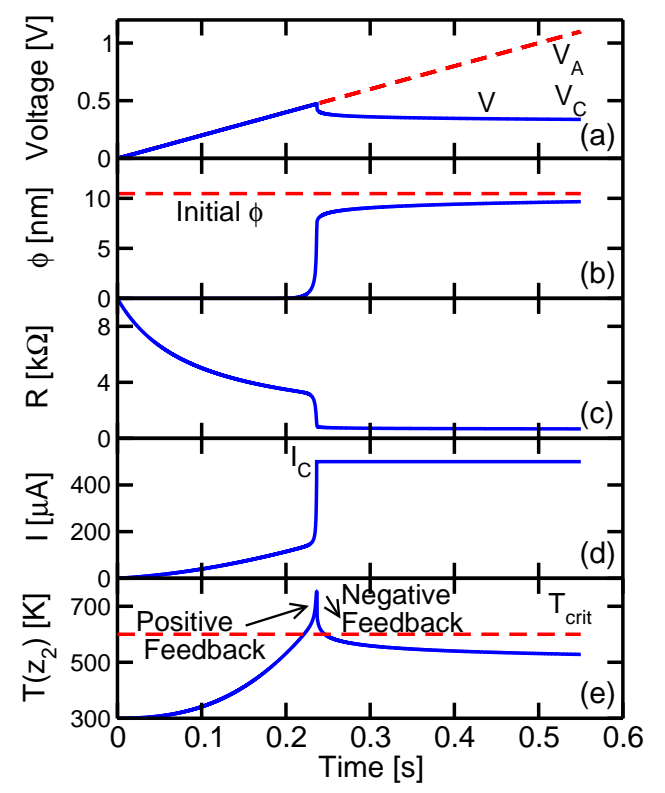

Fig. 7. Calculated voltage $V$, (a), sub-CF diameter $\phi$, (b), resistance $R$, (c), current $I$, (d), and temperature at the $T\left(z_{2}\right)$ edge, (e), as a function of time. The positive feedback is evidenced for $V>V_{\text {set }}$, followed by the negative feedback due to the external $I_{C}$.

Fig. 3, showing the staircase applied voltage $V_{A}$ (a), $\Delta$ (b), the resistance $R$ (c), the current $I$ (d) and $T\left(z_{1}\right)$ (e) as a function of time. The gap length is initially $\Delta=0$ (full set state) at time $t=0$. Application of $V_{A}=0.4 \mathrm{~V}$ causes Joule heating above the critical temperature $T_{\text {crit }} \approx 600 \mathrm{~K}$, defined as the temperature for which the gap growth rate in Eq. (3) reaches $25 \mathrm{~nm} / \mathrm{s}$, thus causing an increase of $\Delta$ (hence $R$ ) and a consequent decrease of $I$. The temperature $T\left(z_{1}\right)$ thus decreases below $T_{\text {crit }}$, thus slowing down of $R$ and $\Delta$. Raising $V_{A}$ to $0.5 \mathrm{~V}$ after $0.5 \mathrm{~s}$ causes a step change of Joule heating above $T_{\text {crit }}$, thus boosting the increase of $\Delta$ and $R$. The consequent decrease of the current, however, again results in a decay of Joule heating, hence of the growth rate. A similar negative-feedback response is seen at the third increase of voltage to $0.6 \mathrm{~V}$ at $1 \mathrm{~s}$. This behavior is at the basis of the gradual resistance increase during the negative $V_{A}$ sweep in Fig. 1b, where the gap length and the resistance stabilize to dynamic values at increasing $V_{A}$.

To validate the model, the experimental and calculated $I$ $V$ curves are shown in Fig. 4 for increasing sweep rate $\beta=d V / d t\left[\mathrm{Vs}^{-1}\right]$, namely $\beta=1 \mathrm{~V}$ (a), $10^{2}$ (b), $10^{4}$ (c) and $10^{6} \mathrm{Vs}^{-1}(\mathrm{~d})$. The parasitic capacitance in our setup was about $10 \mathrm{pF}$, which, multiplied with the matching resistance of $50 \Omega$, results in a RC delay time of less than $1 \mathrm{~ns}$, thus negligible with respect to the maximum $\beta$ in our experiments. Both $V_{\text {reset }}$ and $I_{\text {reset }}=V_{\text {reset }} / R$ increase for increasing $\beta$, as summarized in Fig. 5a and b, respectively, for increasing $I_{C}$. The increase of $V_{\text {reset }}$ and $I_{\text {reset }}$ can be understood by the Arrhenius behavior of growth rate in Eq. (3). As the timescale of the experiment is reduced, the temperature and voltage needed to induce reset must correspondingly increase. The good agreement between calculations and experiments in Figs. 5 and 6 demonstrates the accurate estimation of $E_{A}$
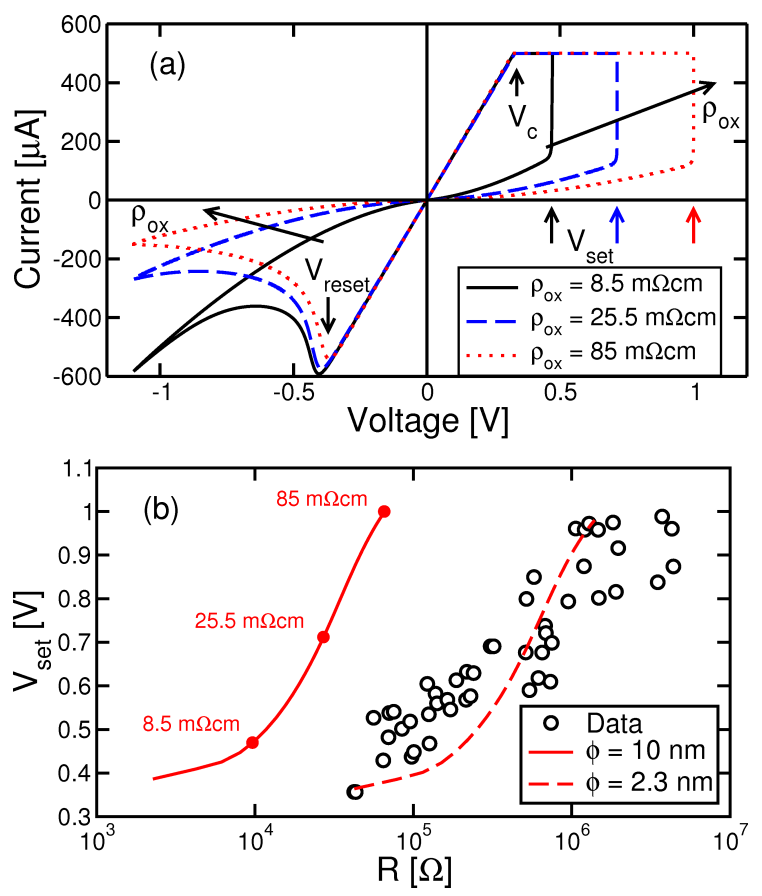

Fig. 8. Calculated $I-V$ curves for RRAM device at increasing $\rho_{o x}$ (a). The leakage current decreases and $V_{\text {set }}$ increases for increasing $\rho_{\text {ox }}$, while $V_{\text {reset }}$ and $V_{C}$ remain constant due to their sole dependence from the CF resistivity. In (b), measured and calculated $V_{\text {set }}$ at increasing resistance of the reset state. Two different $\phi$ CF diameters are shown. Data are obtained from [19].

controlling reset kinetics in Eq. (3). Note that $I_{C}$ controls the size of the CF obtained after set transition, hence $R$ and $I_{\text {reset }}$ [11], while $V_{\text {reset }}$ is independent of $I_{C}$, since the temperature during reset transition only depends on parameters $\rho_{m}$ and $k_{t h, m}[14]$.

\section{B. Set model and simulations}

Fig. 6 schematically shows the set process, starting from the reset state in Fig. 6a, corresponding to the final state in Fig. 2c with an arbitrary gap length $\Delta$. Application of $V_{A}>0$ induces ion migration from the stub at the injecting-electrode side (edge $z_{2}$ ) into the gap, thus resulting in the formation of a sub-filament of diameter $\phi$ (Fig. 6b) and its growth with time (Fig. 6c). The final value of $\phi$ can be smaller than the nominal size of the $\mathrm{CF}$, or it can be higher than that value. As $\phi$ reaches the nominal size of the $\mathrm{CF}$, the whole $\mathrm{CF}$ grows in size as a result of more ion migration from the reservoir. The sub-filament growth rate is modeled by the Arrhenius law:

$$
\frac{d \phi}{d t}=A e^{-\frac{E_{A}}{k T\left(z_{2}\right)}},
$$

where $E_{A}$ is subject to the barrier lowering law of Eq. (4) and the temperature is calculated at the injecting edge $z_{2}$. Fig. 6d shows the equivalent electric circuit used for evaluating $R$, where the gap region resistance is calculated from the parallel contributions of the sub-filament and the oxide resistances.

The calculated $I-V$ curve for set transition can be seen in Fig. 1b, where $R$ decreases abruptly just above the set voltage 
$V_{\text {set }}$, then the voltage across the $\mathrm{CF}$ settles to $V_{C}$ in the current compliance regime due to the $\mathrm{CF}$ growth, where $R$ decreases with time at constant current $I_{C}$. The model accounts for the experimental relationship $V_{\text {set }}>V_{\text {reset }}$, which is due to the different shape of the CF in the full set state (Fig. 2a) compared to the reset state (Fig. 6a). In the latter, the depleted gap causes a relatively low temperature in $z_{2}$, therefore a higher $V$ must be applied to reach the critical temperature $T_{\text {crit }}$.

The abrupt $R$ change at the set transition in Fig. $1 \mathrm{~b}$ can be understood by a positive feedback effect, as described in Fig. 7 showing calculation results for the voltage across the device $V$ (a), the CF diameter $\phi$ (b), the corresponding $R$ (c), the current $I$ (d) and the temperature $T\left(z_{2}\right)$ at the injecting edge (e) during a sweep of the applied voltage $V_{A}$. Initially $\phi$ is equal to zero in the reset state and $R$ decreases for increasing $V$ due to the field effect in Eq. (5). As $T\left(z_{2}\right)$ reaches $T_{\text {crit }}$ by Joule heating at $t \approx 0.23 \mathrm{~s}, \phi$ increases and $R$ decreases steeply due to the growth of the $\mathrm{CF}$ in the gap region. As the sub-filament is formed in the gap, the current (Fig. 7d) and the temperature (Fig. 7e) strongly increase, causing further acceleration of the $\mathrm{CF}$ growth and establishing a positive thermal feedback loop. The voltage across the CF drops in Fig. 7a as the current reaches the compliance current, which thus serves as an external negative feedback for $R$ control during the set transition, which otherwise would obey to an inherent positive feedback. A key parameter for the set transition is $V_{\text {set }}$, marking the condition $T\left(z_{2}\right)=T_{\text {crit }}$ in Fig. 7e. The set voltage $V_{\text {set }}$ was shown to strongly depend on the reset condition, namely deep reset states with high $R$ generally display a large $V_{\text {set }}$ [1], [19], [20]. This is due to a higher $\Delta$ and/or to a lower defect concentration in the gap, resulting in a larger $\rho_{o x}$ in Eq. (5), hence in a lower Joule heating for a given applied voltage. Fig. 8a shows the calculated $I$ - $V$ curves for variable $\rho_{o x}$ in Eq. (5), to describe different gap resistivities thanks to a controlled defect concentration. Three values of $\rho_{o x}$ were assumed, namely $\rho_{o x}=8.5,25.5$ and $85 \mathrm{~m} \Omega \mathrm{cm}$. Changing $\rho_{o x}$ has no impact on $V_{C}, V_{\text {reset }}$ and $I_{\text {reset }}$, since these parameters are controlled by the CF in the set state and not by the gap properties. On the other hand, the increase of $\rho_{\text {ox }}$ causes an increase of the on/off resistance window and of $V_{\text {set }}$ as a result of the reduced Joule heating. Both effects strongly contribute to the operation and reliability of RRAM devices. One of the critical concerns for RRAM, in fact, is the limited resistance window, which makes single-bit and multilevel operation difficult in the presence of programming variability [20], [21] and read noise [22]. A moderate increase of $V_{\text {set }}$ may also reduce read disturb in the reset state, thus improving the read voltage and the corresponding read current. Fig. 8b shows the calculated $V_{\text {set }}$ as a function of $R$ in the reset state, compared to experimental data [19]. Calculations are shown for two values of $\phi$, which can be controlled by $I_{C}$ during the forming operation. Data and calculations show a good agreement for $\phi=2.3 \mathrm{~nm}$. The exponential range covered by the resistance suggests that $R$ is mostly controlled by the defect concentration in the CF gap, which acts on the Fermi level position with respect to the conduction/valence band edges, rather than the gap length $\Delta$ which should impact

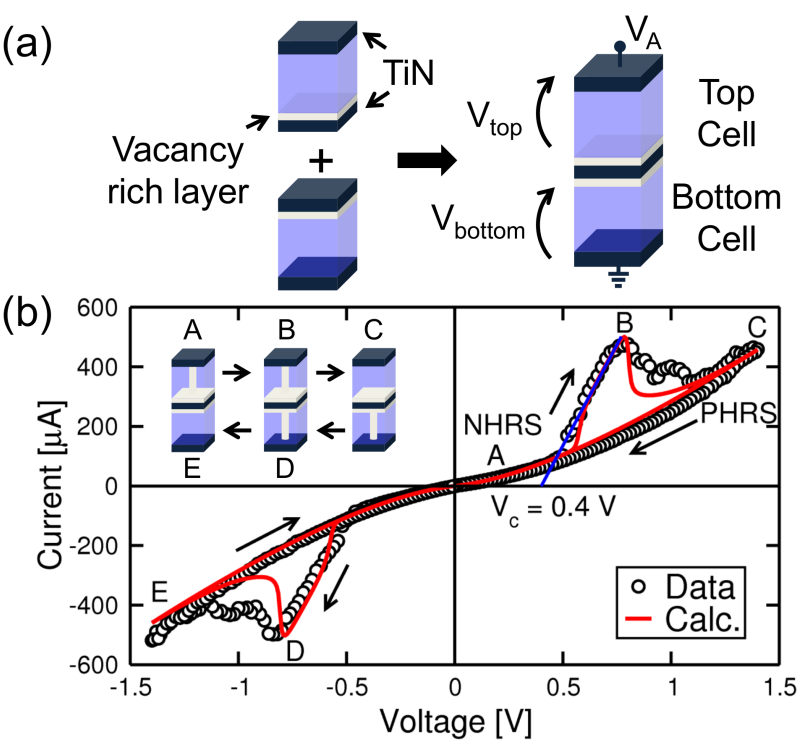

Fig. 9. Schematic structure of the CRS architecture (a). The vacancy-rich side of the asymmetric RRAM stack is shown aligned against the common electrode. In (b), measured, symbols, and calculated, line, $I-V$ curves for CRS. In the inset, application of a positive voltage to the NHRS A results in the set of the bottom cell B, then the reset of the top cell leads to the PHRS C. The symmetric behaviour takes place under negative voltage, leading to the initial NHRS, D and E.

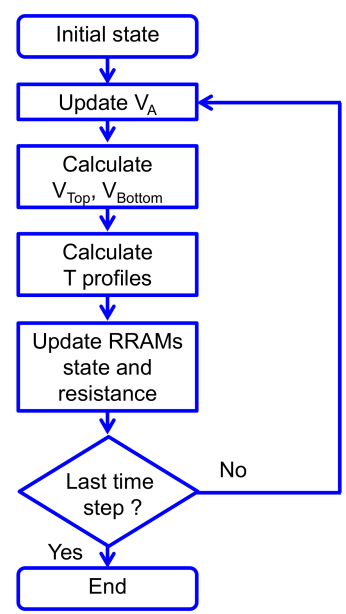

Fig. 10. Flow chart of the numerical model calculation.

only linearly on $R$.

\section{CRS STRUCTURE AND OPERATION}

The analytical model of RRAM can be straightforwardly extended to the complementary resistive switch (CRS) architecture sketched in Fig. 9a. CRS consists of two RRAM with antiserial connection, where the ion-injecting electrodes, i.e., electrodes facing the vacancy-rich layer, are shortcircuited. The CRS is a two-terminal device, thus only the non-injecting electrodes of the top and bottom cells can be accessed by voltage supplies. Note that CRS can also be obtained by shortcircuiting the two non-injecting electrodes of the RRAM cells. Fig. 9b shows the measured $I-V$ curves for a CRS consisting of two antiserially-connected $\mathrm{HfO}_{x}$ RRAM devices 

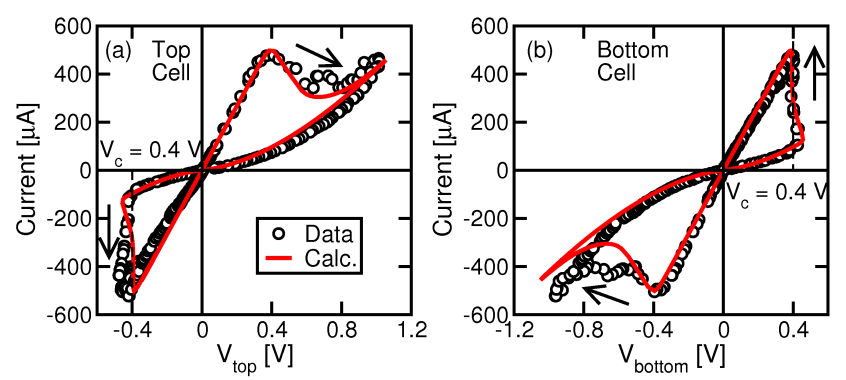

Fig. 11. Measured and calculated $I-V$ curves for top (a) and bottom (b) cells during CRS operation. Data were obtained by connecting a voltage probe to the common electrode during measurement in Fig. 9. The set transition stabilizes at $\mathrm{V}_{C}=0.4 \mathrm{~V}$.
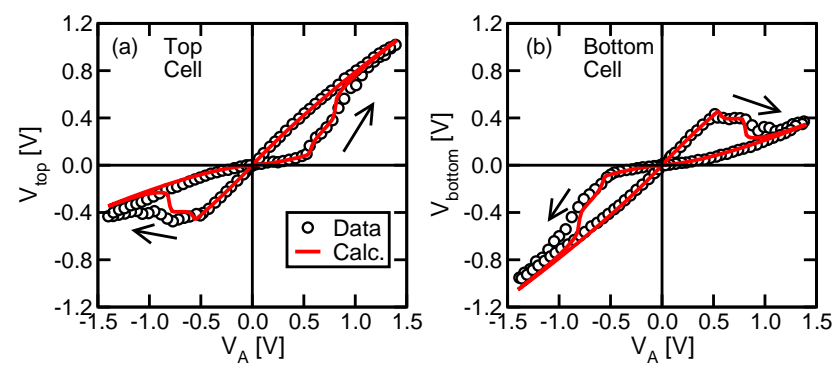

Fig. 12. Measured and calculated voltages $V_{\text {top }}$ (a) and $V_{\text {bottom }}$ (b) as a function of the applied voltage $V_{A}$ during CRS operation. Data were obtained by connecting a voltage probe to the common electrode during measurement in Fig. 9b.

where the ion injecting electrodes were connected together. A voltage $V_{A}$ was applied to the TiN top electrode with the TiN bottom electrode grounded and the common electrode left floating. Initially, the CRS is in state A, where the top RRAM is in the set state and the bottom RRAM is in the reset state, thus the applied $V_{A}>0$ drops almost completely across the bottom RRAM device. As the applied voltage reaches $V_{\text {set }} \approx 0.5 \mathrm{~V}$, the bottom cell undergoes set transition, marking the transition to the low-resistance state B of the CRS with both top and bottom RRAM in the set state. A further increase of the voltage results in a reset transition of the top cell, since the positive voltage applied to the noninjecting electrode drives ion migration from the $\mathrm{CF}$ back into the reservoir of the top cell. The final high-resistance state $\mathrm{C}$ is thus complementary with respect to state $\mathrm{A}$, as the resistance states of the top and bottom cells are exchanged. Application of a negative voltage leads to a similar evolution of the resistance states in the top and bottom RRAM device. First the top cell undergoes set transition, thus achieving the low-resistance state D of the CRS, then reset transition in the bottom device causes the transition to the high-resistance state $\mathrm{E}$, equal to the initial state A. Two different logic states (0 and 1) can thus be stored using two high-resistance states, called negative high-resistance state (NHRS, namely states A or E) and positive high resistance state (PHRS, or state C). Therefore, all the cells always show high resistance, thus preventing any possible sneak path leakage in the memory array and allowing select-less operation [23].

\section{CRS ANALYSIS AND SIMULATIONS}

Fig. 9b also shows the calculated $I-V$ curve of the CRS, obtained by simulating the two RRAM cells with the analytical model. The voltage partition equation was also included, namely:

$$
V_{A}=V_{\text {top }}+V_{\text {bottom }}
$$

where $V_{A}$ is the applied voltage, $V_{t o p}$ is the voltage across the top RRAM and $V_{\text {bottom }}$ is the voltage across the bottom RRAM (see Fig. 9a). The method for calculating the $I$ - $V$ curve of the CRS is shown in the block diagram of Fig. 10. For any simulation time $t_{i}$, the applied voltage $V_{A}$ was partitioned between the top and bottom RRAM based on the cell resistances at the previous simulation time. The voltages $V_{t o p}$ and $V_{\text {bottom }}$ were then used to calculate the current flowing across the cells. At each cycle, the continuity of the current is checked to ensure that the top and bottom current differ by less than $1 \%$. If the current difference is larger, the voltage partition is modified accordingly and the currents are recalculated until the continuity condition is satisfied. The temperature profile at each cell is then calculated, allowing to update the parameters $\phi$ and $\Delta$ by Eqs. (3) and (7) respectively. The cell resistances are then calculated and a new simulation step is started at time $t_{i+1}=t_{i}+\Delta t$. The time interval $\Delta t$ was kept sufficiently small to ensure convergence and accuracy of the simulation. The tolerance of $1 \%$ can be changed according to the required accuracy in the simulation. A parasitic capacitance of about 15 $\mathrm{pF}$ was present at the intermediate node due to the connected probe for monitoring the voltage as in Fig. 12. Multiplying this capacitance with the on-state resistance of about $1 \mathrm{k} \Omega$, we obtain a RC delay time of $15 \mathrm{~ns}$, thus negligible with respect to the maximum sweep rate in our study, namely 1 $\mathrm{V} / \mu \mathrm{s}$.

Fig. 11 shows the measured and calculated $I-V$ curves of the top cell (a) and bottom cell (b), obtained by probing the voltage $V_{\text {bottom }}$ at the intermediate floating electrode while applying a voltage sweep at the CRS in Fig. 9b. Note that the top cell is connected in reverse mode, namely the injecting electrode is negative for positive CRS voltage $V_{A}$, therefore set and reset transitions appear for negative and positive $V_{\text {top }}$, respectively. The $I-V$ curves in Fig. 11 were measured without current compliance, the current in one RRAM being limited by the other RRAM acting as a load resistance. For instance, the set transition at the bottom RRAM takes place at $V_{A}=V_{\text {set }}$ in Fig. 9b, with the top cell in the set state acting as load resistance to limit the current. This results in the voltage snapback at the onset of the set transition of the bottom cell in Fig. 11b. The current then increases almost vertically at constant voltage $V_{\text {bottom }}=V_{C} \approx 0.4 \mathrm{~V}$, due to the voltage driven kinetics of ion migration [11], [24]. A similar characteristic is shown for the top cell in Fig. 11a, although with reverse polarity with respect to the bottom cell. The calculation results account for the $I-V$ curves of top and bottom RRAM cells, as well as for the overall CRS characteristic in Fig. 9b. The constant-voltage set transition at $V_{C}$ is also visible in Fig. $9 \mathrm{~b}$ as a linear $I-V$ curve in the low-resistance state expressed by: 


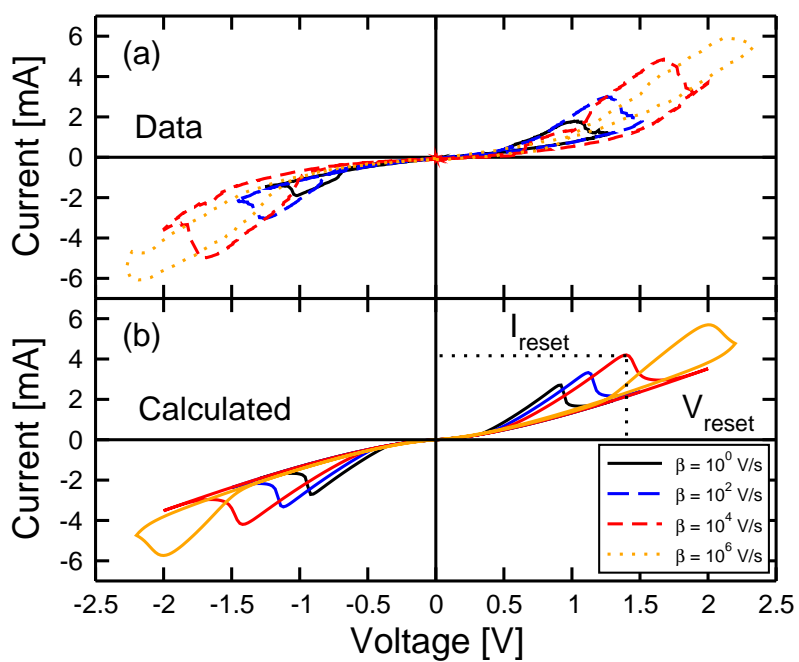

Fig. 13. Measured (a) and calculated (b) CRS $I-V$ curves for increasing sweep rates $\beta=1,10^{2}, 10^{4}$ and $10^{6} \mathrm{Vs}^{-1}$.

$$
I=\frac{V_{A} \pm\left|V_{C}\right|}{R_{L R S}}
$$

where $R_{L R S}$ is the resistance of the CRS with both top and bottom cells in the set state. Plus and minus signs in Eq. (9) apply for negative and positive $V_{A}$, respectively, in Fig. 9b. The extrapolation to zero current in the CRS $I-V$ curve of Fig. 9b allows the extraction of $V_{C}$ [24].

Fig. 12 shows the measured and calculated voltages $V_{t o p}$ (a) and $V_{\text {bottom }}$ (b) as a function of $V_{A}$ along a CRS cycle. During the positive voltage sweep, $V_{\text {top }}$ increases and $V_{\text {bottom }}$ decreases as a result of the set transition in the bottom RRAM at $V_{A} \approx 0.5 \mathrm{~V}$, followed by a further increase of $V_{t o p}$ and a decrease of $V_{\text {bottom }}$ due to the reset transition of the top RRAM at $V_{A} \approx 0.8 \mathrm{~V}$. The opposite transitions take place during the negative $V_{A}$ sweep, where $V_{t o p}$ decreases and $V_{\text {bottom }}$ increases in two steps.

Fig. 13 shows measured (a) and calculated (b) $I-V$ curves for CRS structures at increasing sweep rates $\beta=d V_{A} / d t$, namely $\beta=1,10^{2}, 10^{4}$ and $10^{6} \mathrm{Vs}^{-1}$. The set and reset voltages increase for increasing $\beta$, similarly to the bipolar RRAM behavior in Figs. 4 and 5. The agreement in the figure supports the choice of physical parameters in the model, such as the activation energy, and the Joule heating description.

\section{CRS OPTIMIZATION}

In high-density crossbar arrays based on the CRS concept, low leakage in the high-resistance state should be achieved. However, the PHRS and NHRS in Figs. 9 and 13 display relatively large leakage, due to the relatively low resistance window between set and reset states in oxide-based RRAM devices. The leakage current contributed by all unselected cells in the same bitline of the selected cell might prevent the discrimination between PHRS and NHRS in the CRS array. To optimize the CRS array, the off-state leakage current should be reduced by increasing the resistivity in the CF gap through

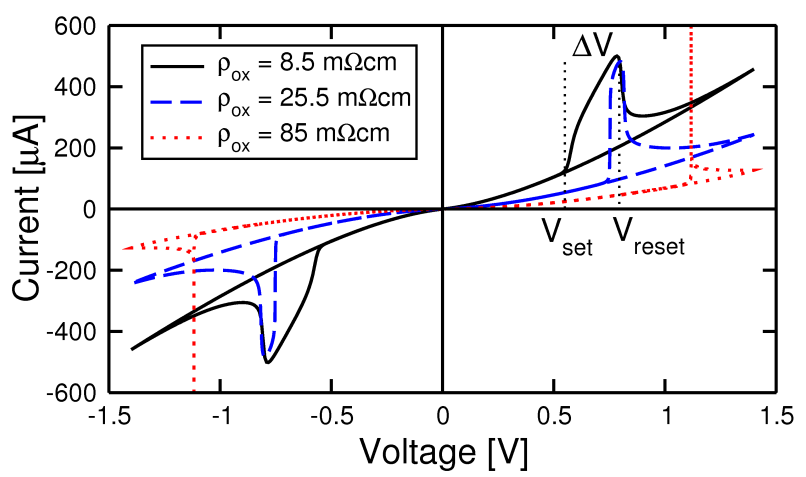

Fig. 14. Calculated $I-V$ curves for the CRS device at increasing $\rho_{o x}$, where the leakage current decrease can be noted. Since $V_{\text {set }}$ increases for increasing $\rho_{\text {ox }}$, the $\Delta \mathrm{V}=V_{\text {reset }}-V_{\text {set }}$ window between set and reset voltages collapses.

a better engineering of the material stack, device process and programming algorithms.

To explore CRS optimization by engineering the gap resistivity, we adopted the bipolar RRAM model and assumed three values of the gap resistivity, namely $\rho_{o x}=8.5,25.5$ and $85 \mathrm{~m} \Omega \mathrm{cm}$ previously considered in Fig. 8. Fig. 14 shows the calculated $I-V$ curves for CRS devices at increasing $\rho_{o x}$ : as $\rho_{o x}$ increases, the resistance of PHRS and NHRS and $V_{\text {set }}$ increase, in agreement with the results of Fig. 8 for a single RRAM device. Other relevant CRS switching parameters, such as $V_{C}$ and $V_{\text {reset }}$, are not significantly affected by $\rho_{o x}$, since these are mostly dictated by $\mathrm{CF}$ electrical/thermal conductivities. The increase of $V_{\text {set }}$ at constant $V_{\text {reset }}$ results in a significant reduction of the read window $\Delta V=V_{\text {reset }}-V_{\text {set }}$, namely the voltage range for the read pulse inducing set in the NHRS and allowing to sense the current $I_{\max }$ in the low-resistance state. As $V_{\text {set }}$ reaches $V_{\text {reset }}$, the read window $\Delta V$ completely vanishes, thus the application of a voltage $V_{A} \geq V_{\text {reset }}$ induces set and reset at the same time (see the $I-V$ curve at $\rho_{o x}=85 \mathrm{~m} \Omega \mathrm{cm}$ in Fig. 14). The increase of $\rho_{o x}$ thus introduces a tradeoff between off-state leakage and read voltage window.

To better evaluate the impact of the $\rho_{o x}$ on the read window, we evaluated the total integrated current during a read triangular pulse from 0 to $V_{\max }>V_{\text {reset }}$, given by:

$$
Q_{\text {read }}=\int_{0}^{t_{\text {read }}} I d t
$$

where $t_{\text {read }}$ is the read pulse width. The charge $Q_{\text {read }}$ can be sensed as the voltage drop at a capacitor used in the read circuit to integrate the current. Note that the read operation induces transition from NHRS to PHRS, thus a negative program operation is needed to recover the PHRS after read [23]. The NHRS and PHRS can be discriminated by $Q_{\text {read }}$, which is expected to be higher in NHRS than in PHRS due to the set transition. However, to discriminate between NHRS and PHRS, $Q_{\text {read }}$ should be significantly higher than the background leakage current from unselected cells. Fig. 15a shows the calculated current response as a function of time during a read pulse with $t_{\text {read }}=100 \mathrm{~ns}$ from 0 $\mathrm{V}$ to $V_{\max }=2.6 \mathrm{~V}$. The comparison between NHRS and PHRS 

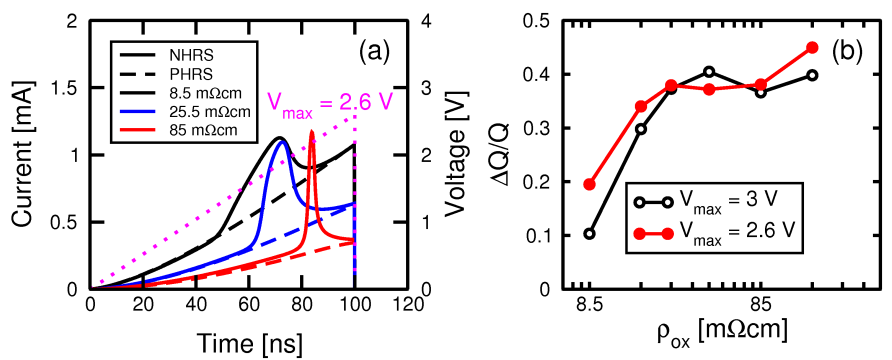

Fig. 15. Calculated current response to a triangular read pulse with amplitude $V_{\max }$, (a), and calculated window $\Delta \mathrm{Q} / \mathrm{Q}$ as a function of $\rho_{o x}$, (b). For increasing $\rho_{o x}$, the relative window saturates, thus giving no additional improvement. Note that readout at $V_{\max }=3 \mathrm{~V}$ leads to a degradation of $\Delta \mathrm{Q} / \mathrm{Q}$, since above $\mathrm{V}=2.6 \mathrm{~V}$ the current only contains off-leakage contribution.

responses shows that, as $\rho_{O x}$ increases, both the background leakage current and the NHRS switching current decrease, the latter being affected by the collapsing $\Delta V$. Fig. 15b shows the calculated $\Delta Q / Q_{\text {read }}$ as a function of $\rho_{o x}$, where $Q_{\text {read }}$ is the integrated charge of the PHRS and $\Delta Q$ is the difference between $Q_{\text {read }}$ of NHRS and PHRS from Eq. (10). For small $\rho_{\text {ox }}, \Delta Q / Q_{\text {read }}$ increases due to the leakage suppression, then it saturates at higher $\rho_{o x}$ due to the collapse of $\Delta V$ affecting the NHRS response. A maximum $\Delta Q / Q_{\text {read }}$ of about $40 \%$ can be achieved, which clearly poses a limit on the maximum size of the CRS crossbar array based on $\mathrm{HfO}_{2}$ RRAM.

\section{CONCLUSIONS}

We presented an analytical model for RRAM switching. The model describes the $\mathrm{CF}$ in terms of the diameter and gap length, which change during set and reset by thermallyactivated ion migration. The model allows to capture the abrupt set transition and gradual reset transition, which can be explained by positive and negative feedback loops between the CF size and the driving forces for ion migration. Finally, the model is used to describe CRS operation and optimization, pointing out the tradeoff between leakage and read window in crossbar arrays.

\section{REFERENCES}

[1] I. G. Baek, M. S. Lee, S. Seo, M. J. Lee, D. H. Seo, D.-S. Suh, J. C. Park, S. O. Park, H. S. Kim, I. K. Yoo, U.-I. Chung, and J. T. Moon, "Highly scalable nonvolatile resistive memory using simple binary oxide driven by asymmetric unipolar voltage pulses", in IEDM Tech. Dig., 2004, pp. 587-590.

[2] R. Waser and M. Aono, "Nanoionics-based resistive switching memories", Nat. Mater., vol. 6, no. 11, pp. 833-840, 2007

[3] H. Y. Lee, P. S. Chen, T. Y. Wu, Y. S. Chen, C. C. Wang, P. J. Tzeng, C. H. Lin, F. Chen, C. H. Lien and M.-J. Tsai, "Low power and high speed bipolar switching with a thin reactive Ti buffer layer in robust $\mathrm{HfO}_{2}$ based RRAM", IEDM Tech. Dig., pp. 297-300, 2008.

[4] P. Huang, X. Y. Liu, W. H. Li, Y. X. Deng, B. Chen, Y. Lu, B. Gao, L. Zeng, K. L. Wei, G. Du, X. Zhang and J. F. Kang, "A physical based analytic model of RRAM operation for circuit simulation", IEDM Tech. Dig., pp. 605-608, 2012.

[5] S. C. Chae, J. S. Lee, S. Kim, S. B. Lee, S. H. Chang, C. Liu, B. Kahng, H. Shin, D.-W. Kim, C. U. Jung, S. Seo, M.-J. Lee and T. W. Noh, "Random Circuit Breaker Network Model for Unipolar Resistance Switching", Adv. Mater., vol. 20, no. 6, pp. 1154-1159, 2008.
[6] S. Yu, X. Guan and H.-S. P. Wong, "On the Switching Parameter Variation of Metal Oxide RRAM-Part II: Model Corroboration and Device Design Strategy", IEEE Trans. Electron Devices, vol. 59, no. 4, pp. 1183-1188, 2012.

[7] S. Menzel, M. Waters, A. Marchewka, U. Boettger, R. Dittmann and R. Waser, "Origin of the ultra-nonlinear switching kinetics in oxide-based resistive switches", Adv. Funct. Mater., vol. 21, no. 23, pp. 4487-4492, 2011.

[8] G. Bersuker, D. C. Gilmer, D. Veksler, P. Kirsch, L. Vandelli, A. Padovani, L. Larcher, K. McKenna, A. Shluger, V. Iglesias, M. Porti, and M. Nafria, "Metal oxide resistive memory switching mechanism based on conductive filament properties", J. Appl. Phys., vol. 110, no. 12, p. 124518, 2011.

[9] R. Degraeve, A. Fantini, S. Clima, B. Govoreanu, L. Goux, Y.-Y Chen, D. J. Wouters, P. Roussel, G. S. Kar, G. Pourtois, S. Cosemans, J. A. Kittl, G. Groeseneken, M. Jurczak and L. Altimime, "Dynamic 'hour glass' model for SET and RESET in $\mathrm{HfO}_{2}$ RRAM", VLSI Tech. Dig., pp. 75-76, 2012

[10] S. Larentis, F. Nardi, S. Balatti, D. C. Gilmer and D. Ielmini, "Resistive Switching by Voltage-Driven Ion Migration in Bipolar RRAM-Part II: Modeling", IEEE Trans. Electron Devices, vol. 59, no. 9, pp.2468-2475, 2012.

[11] D. Ielmini, "Modeling the universal set/reset characteristics of bipolar RRAM by field- and temperature-driven filament growth", IEEE Trans. Electron Devices, vol. 58, no. 12, pp. 4309-4317, 2011.

[12] J. Noh, M. Jo, C. Y. Kang, D. Gilmer, P. Kirsch, J. C. Lee and B. H. Lee, "Development of a Semiempirical Compact Model for DC/AC Cell Operation of $\mathrm{HfO}_{x}$-Based ReRAMs", IEEE Electron Device Lett., vol. 34, no. 9, pp.1133-1135, 2013.

[13] Z. Biolek, D. Biolek and V. Biolkova, "SPICE model of memristor with nonlinear dopant drift", Radioeng., vol. 18, no. 2, pp. 210-214, 2009.

[14] F. Nardi, S. Larentis, S. Balatti and D. Ielmini, "Resistive switching by voltage-driven ion migration in bipolar metal-oxide RRAM-Part I: Experimental study", IEEE Trans. Electron Devices, vol. 59, no. 9, pp. 2461-2467, 2012.

[15] D. C. Gilmer, G. Bersuker, S. Koveshnikov, M. Jo, A. Kalantarian, B. Butcher, R. Geer, Y. Nishi, P. D. Kirsch, and R. Jammy, "Asymmetry, Vacancy Engineering and Mechanism for Bipolar RRAM", International Memory Workshop (IMW), pp. 57-60, 2012.

[16] V. V. Zhirnov, R. K. Cavin, S. Menzel, E. Linn, S. Schmelzer, D. Brauhaus, C. Schindler and R. Waser, "Memory Devices: Energy-SpaceTime Tradeoffs", Proceedings of the IEEE, vol. 98, no. 12, pp. 21852200, 2010.

[17] D. Ielmini and Y. Zhang, "Analytical model for sub-threshold conduction and threshold switching in chalcogenide-based memory devices", J. Appl. Phys., vol. 102, no. 5, pp. 054517-054517-13, 2007.

[18] M. A Panzer, M. Shandalov, J. A. Rowlette, Y. Oshima, Y. W. Chen, P. C. McIntyre and K. E. Goodson, "Thermal Properties of Ultrathin Hafnium Oxide Gate Dielectric Films", IEEE Electron Device Lett., vol. 30, no. 12, pp. 1269-1271, 2009.

[19] S. Koveshnikov, K. Matthews, K. Min, D. C. Gilmer, M. G. Sung, S. Deora, H. F. Li, S. Gausepohl, P. D. Kirsch and R. Jammy, "Real-time study of switching kinetics in integrated $1 \mathrm{~T} / H f \mathrm{O}_{x}$ 1R RRAM: Intrinsic tunability of set/reset voltage and trade-off with switching time", IEDM Tech. Dig., pp. 486-488, 2012.

[20] X. Guan, S. Yu and H.-S. P. Wong, "On the Switching Parameter Variation of Metal-Oxide RRAM-Part I: Physical Modeling and Simulation Methodology", IEEE Trans. Electron Devices, vol. 59, no. 4, pp. 11721182, 2012.

[21] S. Balatti, S. Ambrogio, D. C. Gilmer and D. Ielmini, "Set Variability and Failure Induced by Complementary Switching in Bipolar RRAM", IEEE Electron Device Lett., vol. 34, no. 7, pp. 861-863, 2013.

[22] S. Ambrogio, S. Balatti, A. Cubeta, A. Calderoni, N. Ramaswamy, and D. Ielmini, "Understanding switching variability and random telegraph noise in resistive RAM", IEDM Tech. Dig., pp. 782-785, 2013.

[23] E. Linn, R. Rosezin, C. Kuegeler and R. Waser, "Complementary resistive switches for passive nanocrossbar memories", Nat. Mater., vol. 9, no. 5, pp. 403-306, 2010.

[24] D. J. Wouters, L. Zhang, A. Fantini, R. Degraeve, L. Goux, Y.-Y Chen, B. Govoreanu, G. S. Kar, G. V. Groeseneken and M. Jurczak, "Analysis of Complementary RRAM Switching", IEEE Electron Device Lett., vol. 33, no. 8, pp. 1186-1188, 2012. 